\title{
Temperatura corporal de frangos de corte em diferentes sistemas de climatização ${ }^{1}$
}

\author{
Janaína Schenatto Welker ${ }^{2}$, Alexandre Pires Rosa ${ }^{3}$, Daniella Jorge de Moura ${ }^{4}$, Lizandra \\ Padoin Machado ${ }^{5}$ Fernanda Catelan ${ }^{5}$, Rodrigo Uttpatel ${ }^{5}$
}

\author{
1 Pesquisa financiada pela FAPERGS. \\ 2 Programa de Pós-graduação em Zootecnia da UFSM, Santa Maria, RS, Brasil. \\ ${ }^{3}$ Departamento de Zootecnia da UFSM, CEP: 97105-900, Santa Maria, RS, Brasil. \\ ${ }^{4}$ Departamento de Engenharia Rural, Escola Superior de Agronomia Luiz de Queiroz, Universidade de São Paulo, Piracicaba, SP, Brasil. \\ ${ }^{5}$ Curso de Zootecnia da UFSM, Santa Maria, RS, Brasil.
}

RESUMO - Verificou-se o efeito dos sistemas de climatização de aviários sobre a temperatura média corporal de frangos de corte (TMC). Utilizaram-se 610 fêmeas da linhagem Cobb distribuídas em delineamento de blocos ao acaso repetidos no tempo, considerando o dia como critério de bloqueamento, em esquema fatorial $2 \times 5$, composto de duas orientações solares (Norte-Sul, NS, e Leste-Oeste, LO) e cinco sistemas de climatização (ventilador e nebulização, VN; ventilador com nebulização e sombreamento, VNS; apenas ventilador, V; apenas sombreamento, S; e apenas nebulização, N), de modo que cada tratamento teve sete repetições (dias de coleta). Foram coletados dados meteorológicos para o cálculo de carga térmica de radiação (CTR) e monitoradas as temperaturas de superfície corporal e retal para cálculo da TMC. A associação de ventilação e nebulização melhora significativamente as condições ambientais dos aviários e as condições fisiológicas das aves. Com a utilização adequada de métodos de climatização, é possível a criação de frangos de corte em aviários com orientações solares diversas.

Palavras-chave: ambiência, conforto térmico, frangos de corte, temperatura

\section{Broiler body surface temperature in function of different thermal control systems in broiler housing}

\begin{abstract}
The effect of different thermal control systems on broiler body temperature was evaluated in 610 female broiler chickens, Cobb breed, housed in 10 small scale of aviaries models (treatments). Climate data were collected inside the models and were used to calculate the Thermal Heat Load (THL). Bird surface and rectal temperature were recorded to calculate average body temperature (ABT). Data were always collected from 1:00 and 2:00 pm, the hour of maximum THL inside models. The treatments consisted of a factorial $2 \times 5$ (two orientations and five models of thermal control) and were analyzed as a randomized blocks design, where the days were blocking criteria. Environment and body temperature data were statically analyzed by Duncan test, with $5 \%$ of probability level. The use of forced ventilation associated to misting nozzles improved thermal behavior of the buildings resulting in better broilers physiological conditions. The adequate utilization of thermal control equipments allows to raise broiler chicks in houses with different solar orientation.
\end{abstract}

Key Words: environment, temperature, thermal comfort, broiler chickens

\section{Introdução}

As aves mantêm a temperatura corporal constante quando a temperatura ambiente é termoneutra. Em condições de alta temperatura, os animais precisam de mecanismos físicos, como o resfriamento evaporativo e a redução do consumo de alimentos. Segundo Brown-Brandl et al. (1997), o aumento na temperatura corporal das aves é resultado de um desbalanceamento entre a perda de calor por meios evaporativos e sensíveis e a produção de calor. Quando a temperatura corporal das aves é comparada à temperatura corporal dos mamíferos, aquela, além de ser mais alta, é variável. A temperatura corporal de aves adultas oscila entre 41 e $42^{\circ} \mathrm{C}$. A temperatura do ar na qual este desbalanceamento ocorre varia com a espécie das aves, a idade, a história térmica anterior e a umidade relativa (Brown-Brandl et al., 1997) e ainda com o peso corporal, o sexo, a atividade física e o consumo de alimentos (North \& Bell, 1990). Portanto, o conforto térmico no interior de instalações avícolas é importante, pois condições climáticas inadequadas afetam negativamente o desempenho do animal. Assim, nos climas tropical e subtropical, 
é indispensável o estudo das características ambientais de cada região.

Aves geralmente são desprovidas de glândulas sudoríparas, logo, não têm a capacidade de transpirar; liberam o excesso de calor pela respiração e pelas superfícies desprovidas de penas como cristas, barbelas e área sobre as asas (Carr \& Carter, 1985). Durante períodos de estresse térmico, as extremidades das aves que não possuem penas, como a crista e a barbela ou os pés, normalmente são vasodilatadas (Richards, 1971; Van Kampen, 1971; Nolan et al., 1978; Richards, 1971; Hillman et al., 1982). Van Kampen (1971) calculou que a área total de superfície da crista e barbela das aves corresponde a $10 \%$ da superfície total do corpo, o que reflete a importância dessas regiões corporais na dissipação de calor pelas aves.

Pesquisas comprovam que o aumento da temperatura corporal das aves está relacionado à elevação da temperatura ambiente. A temperatura corporal das aves aumenta quando a temperatura ambiente atinge rapidamente $30^{\circ} \mathrm{C}$ (Boone \& Hughes, 1971). Quando a temperatura eleva gradativamente, a temperatura corporal se mantém constante até a temperatura ambiente atingir $33^{\circ} \mathrm{C}$. Assim como a temperatura, a umidade relativa também afeta a resposta de frangos ao estresse calórico.

Assim, considerando a necessidade de proporcionar aos frangos um ambiente térmico adequado, avaliou-se o efeito de diferentes sistemas de climatização ambiental na temperatura média corporal de frangos de corte.

\section{Material e Métodos}

O trabalho foi conduzido no Laboratório de Avicultura - LAVIC, Departamento de Zootecnia, UFSM - RS, durante o mês de novembro de 2001. Nesse local foram construídos 10 modelos de aviários de alvenaria, cobertos com telhas de barro, com dimensões de 1,40 m × 3,00 m × 1,50 m, construídos em escala reduzida e distorcida de 1:10 na dimensão horizontal e 1:2 na vertical para que representassem instalações avícolas de escala real. Essas dimensões foram definidas em trabalho realizado pela Optima Empresa Júnior do Instituto de Matemática e Ciência da Computação da Universidade Estadual de Campinas, São Paulo, no qual foi determinada a curva isoterma da temperatura no modelo de aviário real.

Foram utilizadas 610 fêmeas da linhagem Cobb com 35 dias de idade, criadas em galpão convencional da empresa Doux-Frangosul, na cidade de Montenegro, Rio Grande do Sul, e transportadas para os modelos onde permaneceram até os 52 dias de idade.

As aves foram distribuídas em delineamento de blocos ao acaso repetidos no tempo, considerando o dia como critério de bloqueamento, em esquema fatorial $2 \times 5$, composto de duas orientações solares (Norte-Sul, NS, e Leste-Oeste, LO) e cinco sistemas de climatização (ventilador enebulização, VN; ventilador com nebulização e sombreamento, VNS; apenas ventilador, V; apenas sombreamento, S; e apenas nebulização, N), no qual cada tratamento teve sete repetições (dias de coleta).

Cada modelo de climatização, mantido com 67 aves (16 aves $/ \mathrm{m}^{2}$ ), foi equipado com um comedouro tubular, um bebedouro, um termômetro de globo negro, um termômetro de máxima e mínima e um higrômetro. As coletas de informações foram realizadas apenas em dias sem precipitação.

Nos modelos Norte-Sul, foram plantadas quatro mudas de árvore em cada lateral do aviário, espaçadas $1,0 \mathrm{~m}$ entre si, de modo que a primeira linha foi plantada a 0,5 m dos modelos para que, nos horários de maior incidência solar direta, a área do telhado fosse sombreada. A segunda linha de árvores foi plantada a 1,0 m dos modelos para sombrear o interior dos aviários, uma vez que, tanto de manhã quanto à tarde, a radiação solar incide diretamente na lateral do modelo. Nos modelos Leste-Oeste, as árvores foram plantadas apenas na lateral norte, considerando o ângulo de inclinação solar no solstício de verão. O sombreamento foi feito por meio do plantio de mudas de uva japonesa (Hovenia dulcis).

A ventilação forçada foi realizada por meio de ventilador com vazão de ar de $1 \mathrm{~m}^{3}$ /segundo. Nos modelos com nebulização, foi instalado, à altura do pé-direito (1,50 m), um bico nebulizador de média pressão com vazão de 5,5 L/hora. A nebulização foi realizada de forma intermitente (cinco minutos ligado e 10 minutos desligado), programada por meio de um timer digital ligado a uma motobomba centrífuga que abastecia o sistema de nebulização.

A avaliação dos parâmetros fisiológicos foi realizada uma vez ao dia, das 13 às $14 \mathrm{~h}$-horários em que a temperatura interna nos modelos é mais alta - utilizando-se uma amostra de $10 \%$ das aves, as quais foram anilhadas para que diariamente fossem utilizadas as mesmas aves e se evitasse o erro amostral. Foram observadas a temperatura de superfície corporal das aves, utilizando-se um termômetro infravermelho (marca Texto, modelo Quiktemp 825-T2), e a temperatura retal (TR), utilizando-se um termômetro digital “tipo espeto" (marca Alla com amplitude de $-50^{\circ} \mathrm{C} \mathrm{a}+150^{\circ} \mathrm{C}$ ).

Os dados fisiológicos coletados foram utilizados no cálculo da temperatura média da pele (TMP) e da temperatura média corporal (TMC) das aves, calculadas de acordo com a equação proposta por Richards (1971), considerando as temperaturas de superfície e a temperatura retal das aves:

$\mathrm{TMP}=(0,03 \mathrm{TC}+0,70 \mathrm{TD}+0,12 \mathrm{TA}+0,06 \mathrm{TCA}+0,09$ $\mathrm{TP}), \mathrm{em}^{\circ} \mathrm{C}$ em que: $\mathrm{TC}=$ temperatura de $\operatorname{crista}\left({ }^{\circ} \mathrm{C}\right) ; \mathrm{TD}=$ 
temperatura do dorso $\left({ }^{\circ} \mathrm{C}\right)$; TA = temperatura da asa $\left({ }^{\circ} \mathrm{C}\right)$; $\mathrm{TCA}=$ temperatura da cabeça $\left({ }^{\circ} \mathrm{C}\right) ; \mathrm{TP}=$ temperatura da pata $\left({ }^{\circ} \mathrm{C}\right)$.

$\mathrm{TMC}=0,3 \mathrm{TMP}+0,7 \mathrm{TR}, \mathrm{em}^{\circ} \mathrm{C}$, em que: $\mathrm{TR}=$ temperatura retal $\left({ }^{\circ} \mathrm{C}\right)$.

As variáveis climáticas coletadas no interior dos modelos foram: temperatura de globo negro (TG), temperaturas máxima e mínima e temperaturas de bulbo seco (TBS) e bulbo úmido (TBU), todas obtidas em equipamentos posicionados a $30 \mathrm{~cm}$ de altura. A umidade relativa no interior dos modelos foi calculada por meio de carta psicrométrica utilizando-se as temperaturas de bulbo seco e bulbo úmido.

Os dados meteorológicos de umidade relativa, temperatura ambiente, velocidade do vento e radiação solar foram monitorados pela Estação Meteorológica da UFSM. Os dados foram coletados quatro vezes ao dia, às 8, 11, 14 e 17 h, considerando o horário oficial de Brasília.

Os dados meteorológicos obtidos no interior dos modelos e a velocidade do vento determinada na Estação Meteorológica da UFSM foram utilizados no cálculo da temperatura média radiante (TMR) e carga térmica de radiação (CTR) incidentes no interior dos modelos, por meio das fórmulas propostas por Bond et al. (1976):

$T M R=100 \times\left[2,51 \times(\sqrt{v v} \times(T G-T B S))+\left(\frac{T g}{100}\right)^{4}\right] 0,25, \mathrm{em} \mathrm{K}$ em que: $\mathrm{TG}=$ temperatura de globo $(\mathrm{K}) ; \mathrm{TBS}$ = temperatura de bulbo seco $(\mathrm{K})$; VV = velocidade do vento $(\mathrm{m} / \mathrm{s})$.

$$
C T R=\sigma \times T M R^{4}
$$

em que: $\sigma=$ constante de Stefan - Boltzman (5,67 * 10-8 W. $\mathrm{K} 4 / \mathrm{m}^{2}$ ).

Os dados de CTR e TMC foram submetidos à análise de variância pelo programa estatístico SAS (1997) e as médias comparadas pelo teste de Duncan (5\%).

\section{Resultados e Discussão}

A carga térmica de radiação (CTR) é um índice físico de conforto que inclui fatores como temperatura, radiação e velocidade do vento, por isso, foi considerada o meio de comparação mais completo entre os modelos; suas médias nos diferentes modelos (Tabela 1), apresentaram diferenças significativas para todos os horários em estudo. Nos horários mais quentes do dia (11 e 14 h), os modelos mais confortáveis termicamente foram o LO-VN e NS-VN, que apresentaram os menores valores de carga térmica de radiação.

O sombreamento, isoladamente, assim como os demais sistemas de climatização, não reduziu a carga térmica de radiação, o que está de acordo com os resultados obtidos por Moura (1998). Em estudos nos quais foram utilizadas árvores mais velhas e com espaçamento menor, foram

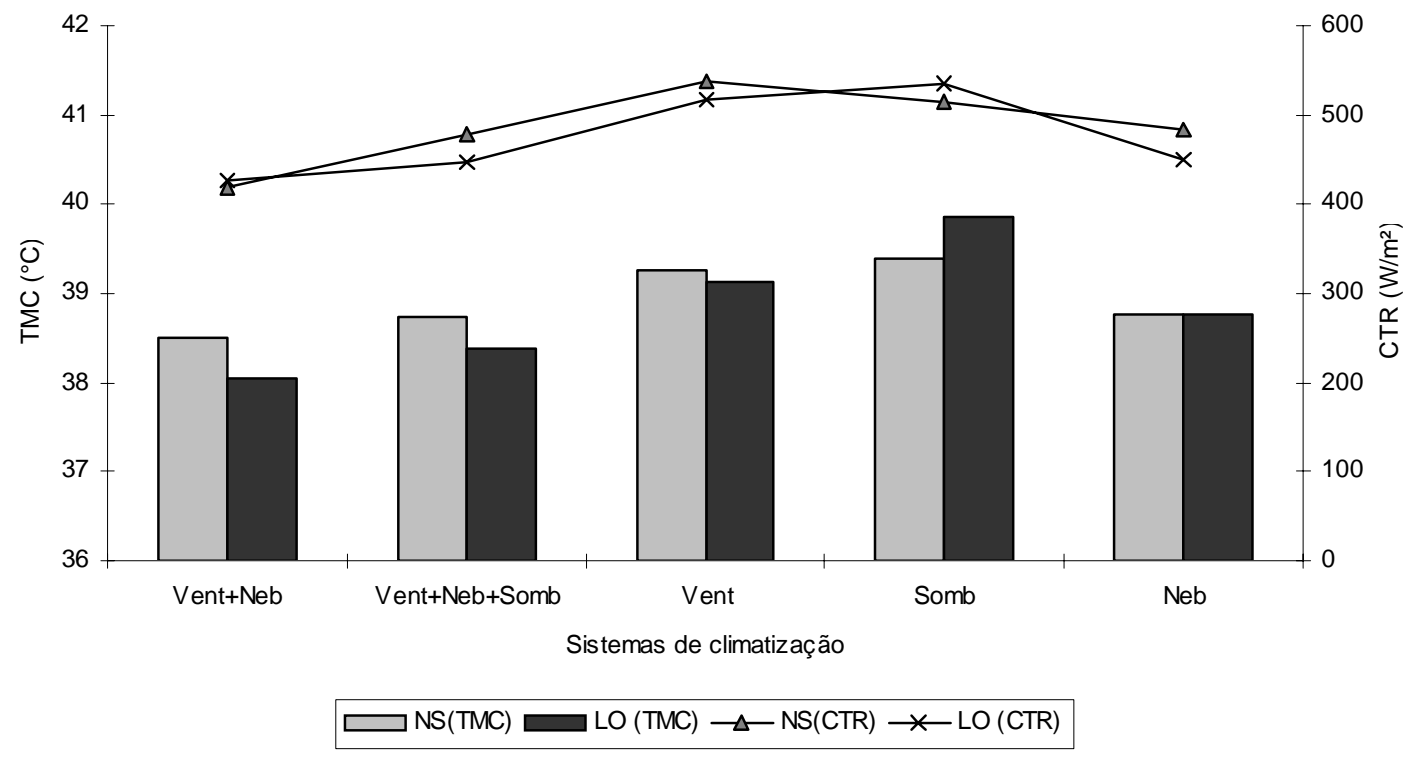

Figura 1 - Temperatura média corporal (TMC) em diversas cargas térmicas de radiação (CTR) em modelos de climatização de aviários. 
Tabela 1 - Carga térmica de radiação (CTR), em W/m², para os diferentes horários de coleta nos sistemas de climatização de aviários

\begin{tabular}{|c|c|c|c|c|}
\hline \multirow[t]{2}{*}{ Análise } & \multicolumn{4}{|c|}{ Horário de coleta de dados } \\
\hline & 8:00 & $11: 00$ & $14: 00$ & $17: 00$ \\
\hline \multicolumn{5}{|l|}{ Orientação solar } \\
\hline Norte-Sul & 521,80 & 484,53 & 467,89 & 471,58 \\
\hline Leste-Oeste & 495,14 & 484,37 & 468,45 & 453,72 \\
\hline $\mathrm{P}$ & 0,0001 & 0,9891 & 0,9604 & 0,2925 \\
\hline \multicolumn{5}{|l|}{ Modelo de climatização } \\
\hline Ventilador e nebulização & 497,73 & 426,39 & 392,14 & 372,41 \\
\hline Ventilador, nebulização e sombra & 500,20 & 462,58 & 437,36 & 453,23 \\
\hline Ventilador & 513,67 & 525,60 & 526,18 & 542,82 \\
\hline Sombra & 515,43 & 536,44 & 533,75 & 515,01 \\
\hline Nebulização & 515,31 & 471,23 & 451,42 & 429,78 \\
\hline$\underline{P}$ & 0,0833 & 0,0001 & 0,0001 & 0,0001 \\
\hline \multicolumn{5}{|l|}{ Modelo } \\
\hline \multicolumn{5}{|l|}{ Norte-Sul } \\
\hline Ventilador e nebulização & $497,58 c$ & $422,35 d$ & $391,47 d$ & 361,69 e \\
\hline Ventilador, nebulização e sombra & $531,86 a$ & $479,34 \mathrm{bcd}$ & $435,37 \mathrm{~cd}$ & 466,56bcd \\
\hline Ventilador & $524,49 a b$ & $522,96 a b c$ & $527,40 a b$ & $577,31 \mathrm{a}$ \\
\hline Sombra & 524,94ab & 523,61abc & $508,23 a b$ & $501,32 a b c$ \\
\hline Nebulização & $530,14 a$ & $474,39 \mathrm{bcd}$ & $476,99 \mathrm{bc}$ & $451,00 \mathrm{bcd}$ \\
\hline \multicolumn{5}{|l|}{ Leste-Oeste } \\
\hline Ventilador e nebulização & $497,88 c$ & $430,43 d$ & $392,82 d$ & 383,13de \\
\hline Ventilador, nebulização e sombra & $468,55 d$ & $445,83 d$ & $439,36 \mathrm{~cd}$ & 439,90cde \\
\hline Ventilador & $502,84 b c$ & $528,25 a b$ & $524,96 a b$ & $508,34 a b c$ \\
\hline Sombra & 505,93abc & $549,26 a$ & $559,27 \mathrm{a}$ & $528,70 \mathrm{ab}$ \\
\hline Nebulização & $500,48 b c$ & $468,07 d$ & $425,85 \mathrm{~cd}$ & 408,55 de \\
\hline $\mathrm{P}$ & 0,0001 & 0,0004 & 0,0001 & 0,0001 \\
\hline Média & 508,47 & 484,45 & 468,17 & 462,65 \\
\hline CV (\%) & 4,36 & 10,21 & 10,09 & 15,19 \\
\hline
\end{tabular}

$a>b>c>d>e(P<0,05)-$ Teste Duncan.

encontrados resultados de sombreamento mais satisfatórios, como no experimento realizado por Silva (1998) com sombreamento intenso de eucaliptos, no qual houve redução de até $11,3 \%$ da TG em aviários de postura, que possilibitou aumento de produtividade de até $12,5 \%$.

Nos modelos Norte-Sul, os maiores valores de TMC foram observados nos modelos com sombreamento (S) e ventilação (V), enquanto nos modelos Leste-Oeste, a maior TMC foi obtida com sombreamento, certamente porque esses modelos apresentavam as piores condições de conforto térmico (Tabela 2). A associação de ventilador e nebulização (VN) promoveu redução da TMC, resultados similares ao obtidos por North \& Bell (1990), Macari et al. (1994) e Cooper \& Washburn (1998). Os modelos que possuíam apenas sombreamento (S) apresentaram as maiores médias de TMC nas duas orientações (Figura 1).

O efeito da ventilação forçada foi maior nos modelos Norte-Sul, o que confirma os resultados obtidos por Moura \& Nääs (1999). Esses autores testaram modelos com simulação do calor total produzido por frangos de corte com 2,0 kg e densidade de 10 aves $/ \mathrm{m}^{2}$, além da
Tabela 2 - Temperatura média corporal (TMC) nos diferentes sistemas de climatização em aviários

\begin{tabular}{ll}
\hline & TMC $\left({ }^{\circ} \mathrm{C}\right)$ \\
\hline Norte-Sul & \\
Ventilador e nebulização & $38,51 \mathrm{~d}$ \\
Ventilador, nebulização e sombra & $38,74 \mathrm{~cd}$ \\
Ventilador & $39,27 \mathrm{~b}$ \\
Sombra & $39,39 \mathrm{~b}$ \\
Nebulização & $38,77 \mathrm{~cd}$ \\
Leste-Oeste & \\
Ventilador e nebulização & $38,05 \mathrm{e}$ \\
Ventilador, nebulização e sombra & $38,39 \mathrm{de}$ \\
Ventilador & $39,13 \mathrm{bc}$ \\
Sombra & $39,86 \mathrm{a}$ \\
Nebulização & $38,76 \mathrm{~cd}$ \\
$\mathrm{P}$ & 0,0291 \\
Média & 38,88 \\
CV $(\%)$ & 1,02 \\
\hline
\end{tabular}

$a>b>c>d>e \quad(P<0,05)$. Teste de Duncan.

presença de lâmpadas no piso, e observaram redução de $10 \mathrm{~W} / \mathrm{m}^{2}$ na carga térmica de radiação atribuída ao efeito da ventilação forçada. Quando utilizado como único método de controle ambiental, o ventilador é capaz de reduzir a 
temperatura média corporal das aves (Figura 1) (Timmons \& Hillman, 1993; Moura, 1998; Nääs et al., 1998).

De modo geral, os valores médios de umidade relativa obtidos nos modelos mantiveram-se dentro do esperado para frangos de corte na fase final de crescimento (75\%). Mesmo os modelos com apenas nebulização não apresentaram umidade relativa maior que $80 \%$, fato relacionado à pequena largura dos modelos (1,4 m), além da efetividade da ventilação natural.

\section{Conclusões}

A associação de ventilação forçada com nebulização influencia positivamente as condições ambientais de aviários e permite a redução da temperatura corporal das aves. A utilização de equipamentos que facilitem a dissipação do calor interno das instalações possibilita a utilização de modelos com orientação Norte-Sul.

\section{Literatura Citada}

BOND, T.E.; KELLY, C.F.; ITTNER, N.R. Radiation studies of painted shade materials. Journal of Agricultural Engineering, v.35, n.6, p.389-392, 1976.

BOONE, M.A.; HUGHES, B.L. Wind velocity as it affects body temperature, water consumption during heat stress of roosters. Poultry Science, v.50, p.1535-1537, 1971.

BROWN-BRANDL, T.M.; BECK, M.M.; SCHULTE, D.D. et al. Physiological responses of tom turkeys to temperature and humidity change with age. Journal of Thermal Biology, v.22, p.43-52, 1997.

CARR, L.; CARTER, T. Housing and management of poultry in hot and cold climates. In: YOUSEF, M.K. (Ed.) Stress physiology in livestock. Boca Raton: CRC Press, 1985. p.74-108.

COOPER, M.A.; WASHBURN, K.W. The relationships of body temperature to weight gain, feed consumption and feed utilization in broilers under heat stress. Poultry Science, v.77, p.237-242, 1998.

HILLMAN, P.E.; SCOTT, N.R.; Van THIENHOVEN, A. Vasomotion in chicken foot: dual innervation of arteriovenous anastomoses. Animal Journal Physiology, v.242, n.1, p.582-590, 1982.

MACARI, M.; FURLAN, R.L.; GONZALES, E. Fisiologia aviária aplicada a frangos de corte. Jaboticabal: FUNEP/UNESP, 1994. 296p.

MOURA, D.J. Avaliação da eficiência térmica de instalações avícolas sombreadas e ventiladas artificialmente, em diferentes orientações. Campinas: Universidade Estadual de Campinas, 1998. 156p Tese (Doutorado em Engenharia Rural). Universidade Estadual de Campinas, 1998.

MOURA, D.J.; NÄÄS, I.A. Avaliação da eficiência térmica de instalações avícolas sombreadas e ventiladas artificialmente, em diferentes orientações no período de verão. Revista Brasileira de Ciência Avícola, v.1, n.3, p.167-174, 1999.

NÄÄS, I.A.; MAGALHÃES, E.S.; CORRIA, M.E. Estudo de comparação das características termohigrométricas na criação de frangos em alta densidade. In: CONFERÊNCIA APINCO DE CIÊNCIA E TECNOLOGIA AVÍCOLA, 1998, Campinas. Anais... Campinas: Fundação Apinco de Ciência e Tecnologia Avícolas, 1998. p.73.

NOLAN, W.F.; WEATHERS, W.W.; STURKIE, P.D. Thermally induced peripheral blood flow changes in chickens. Journal of Applied Physiology, v.44, n.81, p.81-84, 1978.

NORTH, M.O.; BELL, D.P. Commercial chicken production manual. 4.ed. New York: Van Nostrand Reinhold, 1990. 913p.

RICHARDS, S.A. The significance of changes in the temperature of the skin and body core of the chicken in the regulation of heat loss. Journal of physiology, v.216, p.1-10, 1971.

SILVA, I.J.O. Desenvolvimento de modelos matemáticos para análise da influência das condições ambientais na produção de ovos. Campinas: Universidade Estadual de Campinas: Universidade Estadual de Campinas, 1998. 140p. Tese (Doutorado em Engenharia Agrícola) - Universidade Estadual de Campinas, 1998.

TIMMONS, M.B.; HILLMAN, P.E. Partitional heat losses in heat stressed poultry as affect by wind speed. In: LIVESTOCK EnVironment, 1993, Coventry. Anais... [s.n.], 1993. p.265.

Van KAMPEN, M. Some aspects of thermoregulation in the White Legorn fowl. International Journal of Biometeoroly, v.15, p.244, 1971. 